ancestors fabricated no more than 10 years ago. These technological improvements demand corresponding advances in silicon processing at nanometer scale, rendering self-organizing systems as attractive candidates for processes in future IC assembly. Taking the challenge of on-chip capacitor fabrication, a joint research team from IBM T.J. Watson Research Center and the University of Massachusetts has combined a self-organizing diblock-copolymer system with semiconductor processing to produce silicon capacitors with increased charge storage capacity over planar structures. Capacitor design is a difficult task in IC processing since decreasing the physical dimensions results intrinsically in reducing the capacity of storing electrical charge. This issue is fundamental to DRAM cell design, as well as on-chip decoupling capacitors and ferroelectric memories.

As reported in the July 2 issue of Applied Physics Letters, the research team used the diblock-copolymer film as a mask for creating electrode topography, combining the concept of surface roughening (already implemented in DRAM using rugged polycrystalline silicon) with the demonstrated ability of diblock copolymers to be used as masks for dry etching. The choice for materials used was for the diblock copolymers composed of polystyrene (PS) and poly (methyl methacrylate) (PMMA) which, under suitable processing conditions, separate and self-reassemble into a hexagonal lattice of PMMA cylinders in a matrix of PS with a lattice periodicity well below photolithographic resolution limits. Reactive ion etching using this type of mask produced a patterned capacitor electrode with increased surface area, yielding a capacitance increase of $30 \%$ over planar structures. The capacitor dielectric was thermally grown silicon dioxide onto which an aluminum gate electrode was deposited to form a metal oxide semiconductor device. The PS-PMMA diblock copolymer had an average molecular weight of 73,000 $\mathrm{g} / \mathrm{mol}$, polydispersity index of 1.04 , and PMMA weight fraction of $30 \%$. Scanning electron microscope images were used at various steps during the process to confirm its development and control. The hemispherical-shaped capacitors obtained proved to have a $29 \pm 6 \%$ increase in capacitance over planar structures.

The process established by the researchers is compatible with standard semiconductor processing techniques and is scalable to large wafer dimensions. Their results illustrate the potential for selforganizing block-copolymer templates to add functionality to silicon technology.

Claudiu Muntele

\section{Thin-Film Fullerene PV Cell Doubles Previously Achieved Power Conversion Efficiency}

P. Peumans and S.R. Forrest at the Center for Photonics and Optoelectronic Materials at Princeton University have fabricated an organic thin-film photovoltaic (PV) cell with an efficiency nearly twice that of previously fabricated cells. The researchers measured an external power conversion efficiency of $3.6 \pm 0.2 \%$ under AM 1.5 spectral illumination of 1.5 suns on this cell made of copper phthalocyanine $(\mathrm{CuPc})$ and $\mathrm{C}_{60}$. As stated in their article published in the July 2 issue of Applied Physics Letters, "The improved results are primarily a consequence of the long exciton diffusion length of $\mathrm{C}_{60} . "$

The researchers fabricated these novel double heterostructure PV cells in a superstrate configuration using precleaned glass precoated with transparent, conducting indium tin oxide (ITO). The team treated the glass/ITO, which had been solventcleaned, with an $\mathrm{O}_{2}$ plasma and subsequently spin-coated the glass/ITO with a film of 3,4-polyethylenedioxythiophene: polystyrenesulfonate (PEDOT:PSS). Next, they grew the donor-like $\mathrm{CuPc}$, the acceptorlike $\mathrm{C}_{60}$, and then the bathocuproine (BCP) films at room temperature in high vacuum $\left(\sim 1 \times 10^{-6}\right.$ Torr $)$. They deposited the $\mathrm{Al}$ cathode by thermal evaporation.

Since C.W. Tang developed the efficient heterostructure in 1986, researchers investigating thin-film organic PV cells have been making frequent advances, such as the development of the polymer bulk heterostructure donor-acceptor cells in 1995. The team at Princeton had made previous advancements, in part, due to the ability of the $\mathrm{BCP}$ to transmit electrons while blocking exciton recombination at the cathode. However, the incorporation of the fullerene, $\mathrm{C}_{60}$, with a diffusion length of $77 \pm 10 \AA$, instead of other previously used acceptor materials, such as $3,4,9,10$ perylenetetracarboxylic bisbenzimidazole (PTCBI), with a diffusion length of $30 \pm 3 \AA$, is what propelled the efficiency of this cell far beyond the others. The increased diffusion length allowed for enhanced carrier collection across the solar spectrum. While significantly increasing the effi-

\section{Institute for Soldier Nanotechnologies}

The Army announces its intention to create a University Affiliated Research Center (UARC) to develop nanometer-scale science and technology solutions for the soldier. A single university, along with industry partners, will host this center to emphasize revolutionary materials research toward an advanced uniform and protective ensemble concept. In anticipation of the Broad Agency Announcement, the Army will host two pre-competition informational meetings to be held in the August September time frame on the East and West Coast. Registration information, the meeting times and directions to the meeting location are available at the ARO web site:

\section{http://www.aro.army.mil/soldiernano/}

Questions may be posted by contacting QA@arl.aro.army.mil. The questions and answers will be posted at the above web site.

Circle No. 16 on Inside Back Cover 
ciency of these organic PV cells, the researchers found "that materials choice is an important route to cell optimization." Hence, not only has efficiency been improved, but also the understanding of these devices.

PAMELA JOHNSON

\section{The Femtosecond Transient Absorption Signal of UV-Grade Fused Silica Contains Third- and Fifth-Order Nonlinearities}

Femtosecond transient absorption pump-probe spectroscopy and numerical simulations show interference between the third- and fifth-order nonlinear susceptibilities $\left(\chi^{(3)}, \chi^{(5)}\right)$ of ultraviolet (UV)grade fused silica. Katrin Ekvall of the Royal Institute of Technology in Stockholm and Cecilia Lundevall and Peter van der Meulen of Stockholm University have obtained values for the second-order nonlinear refractive index and the three-photon absorption (3 PA) coefficient from $\chi^{(5)}$.

As reported in the June 15 issue of Optics Letters, a compact Ti:sapphire amplified fs laser system produced $\sim 850 \mu \mathrm{J}, 160 \mathrm{fs}$ pulses centered at $775 \mathrm{~nm}$. A 0.2 -mm-thick Type I $\beta$-barium borate second-harmonic generation crystal frequency doubled the fundamental pulse to produce a pump pulse with $\lambda=387.5 \mathrm{~nm}$.

White light continuum probe and reference pulses were obtained by focusing a small fraction of the fundamental pulse in a thin rotating fused-silica plate. Both the third- and fifth-order material responses produce large signals in the detected region.

The intensity of the pump pulse was varied from 90 to 160,210 , and 270 $\mathrm{GW} / \mathrm{cm}^{2}$ by rotating a zero-order halfwave plate in front of the $\beta$-barium borate crystal.

At the lowest intensity, the transient absorption signal was W-shaped. At higher intensities the curve developed an extra dip and the signal increased. The researchers describe the signal as a superposition of three contributions: the real part of $\chi^{(3)}$, dominant at the lowest intensity; a similarly shaped effect with opposite sign from the real part of $\chi^{(5)}$, which produces the dip at higher intensities; and the imaginary part of $\chi^{(5)}$, corresponding to 3 PA.

The researchers numerically solved the probe propagation equation in the presence of third- and fifth-order nonlinearities, obtaining the probe amplitude after propagation through the sample. They calculated the pump-probe signal as a function of probe wavelength and the delay time between the pump and the probe.
A comparison with the experiment gave the real and imaginary parts of $\chi^{(5)}$. The real part is $-5.1( \pm 0.7) \times 10^{-41} \mathrm{~m}^{4} / \mathrm{V}^{4}$, which gives for the second order nonlinear refractive index $-3.3( \pm 0.4) \times 10^{-41} \mathrm{~m}^{4} / \mathrm{V}^{4}$. The error is due to the uncertainty in pump intensity. The imaginary part of $\chi^{(5)}$ is $2.1( \pm 0.3) \times 10^{-41} \mathrm{~m}^{4} / \mathrm{V}^{4}$, which gives for the 3 PA coefficient $5.2( \pm 0.7) \times 10^{-29} \mathrm{~m}^{3} / \mathrm{W}^{2}$. The simulations did not accurately reproduce the measurements at the highest optical pump intensity, indicating the possibility that even higher order processes may play a role in the nonlinear optical response of this glass.

ELIZABETH A. SHACK

\section{Photopatterned Films of Discotic Liquid Crystals Demonstrate Polarized Photoluminescence}

The ability to control the orientation of liquid crystals (LCs) is needed to optimize their properties for use in display devices. As reported in a communication for the May issue of Chemistry of Materials, in a collaborative effort led by Kunihiro Ichimura of the Tokyo Institute of Technology, researchers used a micropatterned film of discotic liquid crystals (DLCs) to demonstrate the first observation of their polarized photoluminescence (PL).

To date, research in the field of DLCs trails that of calamitic liquid crystals (CLCs) due to the relative difficulties of assembling well-defined DLCs. This is due to the high viscosity of DLCs, and their high mesophase temperatures. To overcome these obstacles, the researchers first assembled a thin film that aided orientation, followed by a heating method which aided in the ordering of the liquid crystals (DLCs).

The researchers first assembled a thin film $(25 \mathrm{~nm})$ by spin-coating a $1.0 \mathrm{wt} \%$ solution of poly[4-(4-cyanophenylazo) phenoxyl methacrylate] (A) on a silica plate. This thin film was then subjected to nonpolarized 436-nm light of $3.0 \mathrm{~J} \mathrm{~cm}^{-2}$ fluence with an angle of $45^{\circ}$, which induced photodichroism. Annealing $\mathbf{A}$ at $240^{\circ} \mathrm{C}$ for 30 minutes then enhanced the anisotropy of the film. Micropatterning of the DLCs was achieved by photoirradation through a photomask upon A before the addition of DLCs.

This prepared film aided in the organized assembling of the DLCs. Next, two DLCs were spin-cast onto the thin film A: both pentakis[(4-alkylphenyl)ethyl]benzene derivatives, labeled as DLC-1 and DLC-2. The films were heated and supercooled, which preserved and aided in the orientation of both DLC-1 and DLC-2. A study of the three-dimensional orientation revealed that both DLCs have homeotropic alignments, with angles of $70^{\circ}$ from the surface $\mathbf{A}$. This was demonstrated by monitoring the azimuthal retardation as a function of the angle of incidence of the probe light, which yielded asymmetrical plots.

To further probe the alignment of the DLC films, PL measurements illustrated the outermost DLCs' alignment at a microscopic level. This alignment was found to be an opposite tilt to the actinic light propagation. When DLC-1/A was irradiated under nonpolarized 340-nm excitation at $20^{\circ}$ to the surface normal, distinct s-polarized, and p-polarized PL absorbances were demonstrated. Both intensity peaks were at $\sim 460 \mathrm{~nm}$, despite the nonpolarized excitation. The s-polarized $/ p$-polarized intensity ratio was $\sim 3$. These results show that the 3D ordering of the preliminary A film was transferred to the DLCs' surface.

Also included in this report is an image of the micropatterned DLC films under PL, demonstrating both s-polarized and $p$-polarized PL, while visually demonstrating the propensity for applications in display devices. These DLCs were shown to be stable in ambient conditions for greater than six months. This communication demonstrates an investigation into the orientation of DLCs via PL, and a possible fabrication technique that results in better control of such orientation. Better orientational control of DLCs will enhance the performance of DLC-based devices such as thin film displays.

MATHEW M. MAYE

\section{$\mathrm{SrTiO}_{3}$ Single Crystals Achieve 8\% Plastic Strain When Tested in Compression at Room Temperature}

Ceramic oxides tend to fail mainly by brittle fracture at ambient or lowtemperatures, due to the ionic and covalent nature of the atomic bonding. However, a group of researchers from the Max Planck Institute in Stuttgart has obtained plastic deformation in compression from $\mathrm{SrTiO}_{3}$, a ceramic oxide with a perovskite structure. The researchers observed a ductile-brittle-ductile transition when testing in a range of temperatures from $78 \mathrm{~K}$ to $1800 \mathrm{~K}$, as reported in the May issue of the Journal of the American Ceramic Society. The samples were ductile in the temperature ranges from $78 \mathrm{~K}$ to $1050 \mathrm{~K}$ and between 1500 and $1800 \mathrm{~K}$, and brittle between $1050 \mathrm{~K}$ and $1500 \mathrm{~K}$.

In order to gather basic information about the nature of the deformation mechanism on this material, the researchers tested single crystals of $\mathrm{SrTiO}_{3}$ oriented with the $\langle 001\rangle$ axis parallel to the compression axis. Tests were conducted in air 